\title{
Reformbedarf der ambulanten Bedarfsplanung
}

\begin{abstract}
Unstreitig besteht ein Reformbedarf der ambulanten Bedarfsplanung. Die starre Ausrichtung der Bedarfsplanung an dem Stichtag 31.12.1990 und die dem Schutz der Freiberuflichkeit dienende Abschaffung gegenüber dem Versorungsangebot insbesondere der Krankenhäuser wird so den Anforderungen an eine stärker regionalisierte und sektorenübergreifend ausgerichtete Versorgungsplanung nicht mehr gerecht. Der Artikel zeigt Probleme, Lösungsansätze und eigene Vorstellungen auf.
\end{abstract}

\section{Rainer Hess}

\section{Mängel der bestehenden vertragsärztlichen Bedarfsplanung}

Die bestehenden Bedarfsplanungsrichtlinien sind nicht mehr zeitgemäß. In dieser Feststellung sind sich alle Beteiligten im Unterausschuss Bedarfsplanung des G-BA einig. Auch in der Begründung dieser Feststellung besteht weitgehender Konsens:

1.1 Die gesetzlich auf den Stand vom 31.12.1990 festgeschriebenen Verhältniszahlen zwischen den Zahlen der Ärzte und denen der Bevölkerung erfüllen nicht mehr den Zweck, den allgemeinen bedarfsgerechten Versorgungsgrad arztgruppenbezogen und als Grundlage für Planungsentscheidungen abzubilden. Die Einführung eines diese Verhältniszahlen korrigierenden Demographiefaktors durch Beschluss des G-BA vom 15. 7. 2010 berücksichtigt jetzt zwar die sich auf die Morbidität auswirkende Alterung der Bevölkerung. Dies geschieht aber nur bei einer den Fachgruppendurchschnitt deutlich überschreitenden Behandlungsfallzahl und einer daraus abgeleiteten erhöhten Morbidität der Bevölkerung.

1.2 Die in den Bedarfsplanungsrichtlinien abgebildete Arztgruppenstruktur ist zu grob; sie bildet die seit ihrer Einführung erfolgte Spezialisierung nicht ab. Insbesondere ist sie völlig ungeeignet für die in § 87 Abs. 2e SGB V ab 1. 1. 2010 vorgesehene ökonomische Steuerung von Über- und Unterversorgung durch Zu- und Abschläge zum Orientierungspunktwert.

1.3 Der Faktor, mit dem Ärzte und Psychotherapeuten auf den allgemeinen Versorgungsgrad ihrer Arzt-

Dr. Rainer Hess ist unparteiischer Vorsitzender des Gemeinsamen Bundesausschusses in Berlin gruppe angerechnet werden, ist mit 1 oder 0,5 bzw. 1, 0,75, 0,5, 0,25 bei angestellten Ärzten ausschließlich auf den Vertragsarztsitz bezogen und berücksichtigt nicht die Organisationsstruktur (MVZ, Berufsausübungsgemeinschaft) und eine Tätigkeit an weiteren Orten.

1.4 Die Bedarfsplanung selbst ist auf die an Stadt- und Landkreisen ausgerichteten Planungsbereiche bezogen ohne systematisierte Berücksichtigung der lokalen Versorgungsstrukturen.

1.5 Sonderbedarfszulassungen zur Berücksichtigung zunehmend spezialisierter Versorgungsangebote erfolgen nicht nach systematisierten Kriterien und berücksichtigen nicht ausreichend das Angebot der stationären Versorgung.

Die vertragsärztliche Bedarfsplanung garantiert zwar in der Regelversorgung zumindest für die fachärztliche Versorgung noch eine flächendeckende kassenübergreifende Versorgung mit niedergelassenen Ärzten; sie bindet aber die Struktur und die Qualität des regionalen Versorgungsangebotes nur unzureichend ein. Im Vertragswettbewerb reduziert sie sich zunehmend auf die Festlegung derjenigen Vertragsärzte und Vertragspsychotherapeuten, mit denen die einzelne Krankenkasse Verträge abschließen darf (oder muss). Dabei lässt die nach Landesrecht erfolgende Krankenhausplanung für die Krankenkassen überhaupt keinen Vertragsspielraum zu.

\section{2. Änderungsvorschläge}

Im Unterausschuss Bedarfsplanung wird seit 2009 über eine Novellierung der Bedarfsplanungsrichtlinie insbesondere im Hinblick auf die damals anstehende Einführung einer vergütungsrechtlichen Steuerung von Über- und Unterversorgung beraten. Grundsätzliche Übereinstimmung besteht zu der Notwendigkeit einer stärkeren Regionali- 
sierung in der Anwendung der nach wie vor bundeseinheitlich vorzugebenden Planungskriterien und in einer gesonderten Behandlung der hoch spezialisierten Versorgung mit dem Ziel einer stärker sektorenübergreifenden Ausrichtung. Diskutiert wird über eine Anpassung der Verhältniszahlen auf den Stand 2010 unter stärkerer Differenzierung der Arztgruppen, eine Definition von drei Versorgungsbereichen: hausärztliche-fachärztliche-spezialisierte fachärztliche Versorgung ( $\S 115 b, 116 b$, Schwerpunkte der WBO), Einführung des von der KBV entwickelten Moduls einer kleinräumigen Bedarfsplanung, jedenfalls für die hausärztliche Versorgung, Bildung unterschiedlich großer Planungsbereiche für die fachärztliche Versorgung und Planung der hochspezialisierten Versorgung auf KV-Ebene. Es gibt aber grundlegende Differenzen über die politische Ausrichtung der Neuordnung einer Bedarfsplanung. Deswegen ist eine konsentierte Vorlage einer neuen Bedarfsplanungsrichtlinie nicht zu erwarten.

2.1 Der GKV-Spitzenverband akzeptiert eine stärkere Regionalisierung der Bedarfsplanung mit lokal zu erwartenden zusätzlichen Arztsitzen nur Zug um Zug gegen den Abbau von Überkapazitäten und stellt die Entschädigungspflicht für die Nichtausschreibung einer Praxisnachfolge grundsätzlich in Frage.

2.2 Die KBV sieht die Beseitigung einer aus ihrer Sicht bereits bestehenden oder drohenden Unterversorgung als Schwerpunkt einer Neuordnung der Bedarfsplanung. Sie will deswegen die arztgruppenbezogenen Verhältniszahlen als „Relikt einer Überversorgungsplanung " abschaffen und durch eine bedarfsgerecht mit Hilfe ihres Moduls der kleinräumigen Bedarfsplanung erfolgte Festlegung von Versorgungssitzen ablösen, für die in der Zukunft nur noch eine Vertragszulassung erfolgen kann. Für den damit verbundenen Abbau nicht bedarfsgerechter Vertragsarztsitze ist eine Entschädigungsregelung vorgesehen. Da die Umsetzung dieses Reformkonzeptes eines grundlegend neuen Gesetzesauftrages bedarf, wendet sich die KBV gegen eine Weiterentwicklung der Bedarfsplanungsrichtlinie auf der jetzigen Rechtsgrundlage.

2.3 KBV und DKG sind sich einig, dass für die hochspezialisierte Versorgung eine eigenständige sektorenübergreifende und stärker wettbewerblich ausgerichtete Regelung erfolgen soll und ein Koordinierungsgremium auf der Landesebene hierfür gebildet werden soll. Die dafür aus Sicht der DKG erforderliche Herausnahme der spezialisierten Versorgung aus der vertragsärztlichen Bedarfsplanung und die Öffnung der Krankenhäuser für die spezialisierte fachärztliche Versorgung, mit ausschließlicher Steuerung über Anforderungen an die Qualitätssicherung, wird aber weder von der KBV noch vom GKV-Spitzenverband mitgetragen.

2.4 Die Patientenvertreter im G-BA fordern eine baldmögliche Novellierung der Bedarfsplanungsrichtlinie, um die aus ihrer Sicht bestehenden gravierenden Mängel in der jetzigen Planung abzustellen.
Von ihnen wird insbesondere die Neuausrichtung der Verhältniszahlen und Arztgruppen mit der Zielsetzung einer stärkeren Differenzierung des Versorgungsangebotes und dessen Berücksichtigung im Rahmen einer regionalisierten Bedarfsplanung angemahnt.

2.5 Im G-BA erörtert wurden auch die von der GMK geforderten Gesetzesänderungen zur Stärkung der Gestaltungsmöglichkeiten der Länder in der medizinischen Versorgung, soweit sie sich auf die vertragsärztliche Bedarfsplanung beziehen. Auch diesen Vorschlägen liegt eine stärkere Regionalisierung und sektorenübergreifende Ausrichtung der Bedarfsplanung zugrunde. Sie wird verbunden mit der Forderung nach Mitwirkung von Vertretern der Länder an den Beratungen des G-BA zur Bedarfsplanung und der Einrichtung eines sektorenübergreifenden Koordinierungsgremiums auf der Landesebene

\section{Weiterentwicklung der Bedarfsplanung}

Im Unterausschuss des G-BA wurde vor dem Hintergrund dieser sehr komplexen Ausgangslage für die in 2011 vom BMG angekündigten Beratungen ebenfalls kontrovers ein Richtlinienentwurf des UA-Vorsitzenden diskutiert, der aufzeigen soll, welche Möglichkeit das geltende Recht zur Weiterentwicklung der Bedarfsplanungsrichtlinie bietet. Im Umkehrschluss ergibt sich daraus auch, welche Regelungen ohne eine Gesetzesänderung nicht möglich sind (einseitiger Abbau von Überkapazitäten, Ausgliederung der hoch spezialisierten Versorgung aus der vertragsärztlichen Bedarfsplanung oder Einbeziehung der ambulanten Behandlung durch Krankenhäuser nach § 116b SGB V in die Bedarfsplanung). Eine systemimmanente Anpassung der Bedarfsplanungsrichtlinie an die jetzigen Erfordernisse lässt sich aber durch folgende Änderungen erreichen:

\subsection{Neuausrichtung der Verhältniszahlen (2010) und der Arztgruppen}

§ 101 Abs. 2 S. 1 SGB V erlaubt eine Festlegung neuer Verhältniszahlen, wenn dies wegen einer Änderung der fachlichen Ordnung der Arztgruppen oder zur Sicherstellung der bedarfsgerechten Versorgung erforderlich ist. Damit eröffnet das geltende Recht die Möglichkeit, sowohl die Arztgruppen insbesondere an Entwicklungen des Weiterbildungsrechts (zB Aufgliederung der Arztgruppe der Nervenärzte in Neurologen und Psychiater; Ausgliederung von Schwerpunkten der Fachgebiete als Teil einer gesondert zu bewertenden hochspezialisierten Versorgung) anzupassen und grundsätzlich die Verhältniszahlen bedarfsgerecht neu festzusetzen. Um bei einer Neuberechnung der Verhältniszahlen zum Versorgungsstand 2010 statistische Verwerfungen auszuschließen, bedarf es einer Adjustierung am bestehenden Versorgungsgrad. Hierfür soll ein Zeitraum von zwei Jahren bis 2012 dienen, in dem die bisherigen Verhältniszahlen als Grundlage für 
Zulassungsentscheidungen der Zulassungsausschüsse zunächst weitergelten.

\subsection{Veränderung der Planungsbereiche}

Die regionalen Planungsbereiche sollen nach $\S 101$ Abs. 1 S.6 SGB V den Stadt und Landkreisen entsprechen. Dies lässt mit entsprechender Begründung Abweichungen zu, die es bereits jetzt mit der Beibehaltung „alter" Planungsbereiche bei Verwaltungsreformen gibt, die bestehende Landkreise und Städte zu größeren Regionen zusammenfassen. Eine Regionalisierung der Bedarfsplanung auf die kommunale Ebene von Gemeinden und Ortschaften würde selbst für die hausärztliche Versorgung sich überschneidende Versorgungsströme gesondert erfassen müssen. Kleinräumige Bedarfsplanung sollte daher nicht zu kleinräumigen Planungsbereichen führen, sondern innerhalb der grundsätzlich fortbestehenden Ausrichtung der Planungsbereiche an den Stadt- und Landkreisen eine kleinräumige Versorgungsanalyse mit dem dazu entwickelten Modul der KBV ermöglichen, deren Konsequenzen für die Bedarfsplanung mit dem Instrument des lokalen Versorgungsbedarfs gezogen werden können. Für die hoch spezialisierte Versorgung kann abweichend hiervon die KV-Ebene als Planungsbereich in den Richtlinien festgelegt werden. Auch für Fachgebiete mit einem größeren Einzugsgebiet können größere Planungsbereiche in den Richtlinien definiert werden (z.B. Landkreis(e) mit eingeschlossener kreisfreier Stadt).

\subsection{Lokaler Versorgungsbedarf}

Den Landesausschüssen der Ärzte und Krankenkassen obliegt nach Maßgabe der Bedarfsplanungsrichtlinien des G-BA die Feststellung, dass in einem nicht unterversorgten Planungsbereich zusätzlicher lokaler Versorgungsbedarf besteht ( $\$ 100$ Abs. 3 SGB V). Die Bedarfsplanungsrichtlinien des G-BA haben nach § 101 Abs. 1 S. 1 Nr. 3a SGB V die allgemeinen Voraussetzungen festzulegen, nach denen die Landesausschüsse einen solchen lokalen Versorgungsbedarf feststellen können. Dieses bereits im SGB V enthaltene Instrument sollte zur Regionalisierung der Bedarfsplanung genutzt werden. Zu diesem Zwecke muss das von der KBV entwickelte und ggf. weiterzuentwickelnde Modul der kleinräumigen Bedarfsplanung rechtsverbindlich für die Landesausschüsse zur Erstellung von Versorgungsanalysen in den Planungsbereichen vorgegeben werden. Die Prüfung des lokalen Versorgungsbedarfes ist insbesondere auf folgende Versorgungsaspekte auszurichten:

3.3.1 Besteht im Planungsbereich und in den Bezugsregionen ein ausreichendes Angebot an haus- und fachärztlicher Versorgung unter Berücksichtigung der jeweils zumutbaren Entfernungen, der auf Grund der regionalen Infrastruktur (Wohngebiete, Industriegebiete, Pendler, Verkehrsverbindungen etc.) zu erwartenden Inanspruchnahme ärztlicher Leistungen, jeweils differenziert nach den fachli- chen Ausrichtungen der Ärzte und den Alters- Geschlechts und Morbiditätsstrukturen der von ihnen zu versorgenden Bevölkerung?

3.3.2 Umfasst dieses Angebot auch die erforderlichen präventiven, kurativen, rehabilitativen und palliativen Leistungen? Stehen im ausreichenden Umfang pflegerische Leistungen, andere gesundheitliche Leistungen und soziale Dienste zur Verfügung?

3.3.3 Bestehen ergänzende Angebote ambulanter Leistungen durch Krankenhäuser oder Reha-Einrichtungen, Pflegeeinrichtungen, Hospize etc., und wie werden sie genutzt?

3.3.4 Welche Entwicklung des medizinischen Versorgungsbedarfs- und des Versorgungsangbebotes ist bei der Wohnbevölkerung ihrer Zahl, ihrer Altersstruktur, ihrer Nachfrage nach ärztlichen Leistungen (Alter, Geschlecht, Morbidität) und bei den Ärzten ( deren Tätigkeitsgebiet, Altersstruktur (inklusive des Abgabealters und der zu erwartenden Neuzugänge)), und den anderen Gesundheitsberufen zu erwarten?

Der Landesausschuss stellt auf der Grundlage der Überprüfung eines zusätzlichen lokalen Versorgungsbedarfes soweit als möglich fest, welche Anforderungen an die Strukturen der vertragsärztlichen Versorgung im jeweiligen Planungsbereich und in den Bezugsregionen unter Berücksichtigung der jeweiligen Infrastruktur und deren zu erwartender Entwicklung zu stellen sind, welche Vertragsarztsitze diesen Anforderungen auch in ihrer Organisationsstruktur am ehesten entsprechen und gegebenenfalls, welcher zusätzliche lokale Versorgungsbedarf besteht.

Auf der Grundlage der Versorgungsanalyse trifft der Landesausschuss Feststellungen zu einem zusätzlichen lokalen Versorgungsbedarf häufig unter gleichzeitiger Feststellung von Überkapazitäten in den Zentren des betreffenden Planungsbereiches. Zur Deckung eines zusätzlichen Versorgungsbedarf sind dann zunächst die gesetzlich bestehenden Möglichkeiten einer Übernahme der Versorgung durch bestehende Vertragsarztsitze und Ermächtigungen als Grundlage für Entscheidungen des Zulassungsausschusses und der Kassenärztlichen Vereinigung aufzuzeigen (Umschreibung von Vertragsarztsitzen, Genehmigung weiterer Orte zur Ausübung der vertragsärztlichen Tätigkeit auch mit angestellten Ärzten, Nachfolgebesetzung nach § 103 Abs. 4 SGB V mit entsprechenden Auflagen, Erweiterung bestehender Ermächtigungen oder Neuerteilung beantragter Ermächtigungen zur Teilnahme an der vertragsärztlichen Versorgung etc.). Die Ausschreibung zusätzlicher Vertragsarztsitze darf durch den Landesausschuss erst freigegeben werden, wenn sich der zusätzliche Versorgungsbedarf nicht durch vorgenannte Maßnahmen decken lässt.

\subsection{Sonderbedarfszulassung}

Nach § 101 Abs. 1 S. 1 Nr. 3 SGB V haben die Bedarfsplanungsrichtlinien des G-BA Vorgaben für die ausnahmsweise Besetzung zusätzlicher Vertragsarzte 
zu treffen, soweit diese zur Wahrung der Qualität der vertragsärztlichen Versorgung in einem Versorgungsbereich unerlässlich sind. Abschnitt 24 der RL enthält auf dieser Grundlage Voraussetzungen für „Sonderbedarfszulassungen“ die insbesondere Bereiche der hoch spezialisierte Versorgung betreffen. Das Instrument der Sonderbedarfszulassung kann daher dazu genutzt werden, das Problem einer sektorenübergreifenden Bedarfsplanung mit den Mitteln des geltenden Rechts einer Lösung zuzuführen, die allerdings an die unter 3. aufgezeigten Grenzen stößt. Um über das Instrument der Sonderbedarfszulassung für den Bereich der hoch spezialisierten Versorgung zunächst sektorenüberschreitende Versorgungsanalysen durchführen zu können, bedarf es einer gesonderten Erfassung des Versorgungsangebotes derjenigen Vertragsärzte, die spezialistische Versorgungsaufgaben wahrnehmen. Dies sind insbesondere Zusammenschlüsse von Ärzten (Schwerpunktpraxen, OP-Zentren, Dialyseeinrichtungen, Großgerätebetreiber) in Form von Berufsausübungsgemeinschaften oder MVZ aus Fachärzten mit operativer Ausrichtung oder Schwerpunktkompetenzen der Weiterbildungsordnung sowie einer Ausrichtung auf die spezialisierten Facharztbezeichnungen der Chirurgie und inneren Medizin, Fachärzte für Stimm-, Sprech- und Sprachstörungen und Humangenetiker.

3.4.1 Die Versorgungsanalyse für diesen spezialistischen Versorgungsbedarf sollte auf der KV-Ebene unter Einbeziehung entsprechender Versorgungsangebote anderer Einrichtungen, insbesondere Krankenhäuser erfolgen. Letzteres gilt insbesondere für das Versorgungsangebot von Krankenhäusern zur ambulanten Behandlung nach § 116b SGB V oder zur Durchführung ambulanter Operationen und stationsersetzender Eingriffe nach $\S 115 b$ SGB V.

3.4.2 Zur Deckung eines spezialistischen Versorgungsbedarfs sind vom Landesausschuss - soweit möglich - in Abstimmung mit den in Frage kommenden Einrichtungen unter Beteiligung von Vertretern der für die Krankenhausplanung zuständigen Stellen als Grundlage für vom Zulassungsausschuss zu treffende Zulassungsentscheidungen sektorenübergreifende Kooperationsmöglichkeiten aufzuzeigen. Stehen derartige sektorenübergreifende Kooperationsmöglichkeiten zur Verfügung, besteht grundsätzlich kein Bedarf für eine Zulassung zusätzlicher Vertragsarztsitze. Die Kooperation ist dann über die bestehenden Instrumente der Teilzulassung, Ermächtigung, MVZ - Zulassung, Genehmigung weiterer Orte für die vertragsärztliche Tätigkeit, Konsiliararzttätigkeit, Belegarztanerkennung, Praxisklinikbildung, oder über Kooperationsverträge zur gemeinsamen Gerätenutzen etc. zu bilden.

3.4.3 Eine die vertragsärztliche Bedarfsplanung und die Krankenhausplanung rechtlich zusammenführende Bedarfsplanung würde voraussetzen, dass entweder der Bund auf seine konkurrierende Gesetzgebung verzichtet und den Ländern die Bedarfsplanung insgesamt überlässt oder über eine Grundgesetzänderung die Gesetzgebungskompetenz des Bundes für die Krankenhausplanung begründet wird. Beides ist nicht zu erwarten. Die ausschließliche Gesetzgebungskompetenz der Länder für die Krankenhausbedarfsplanung (Landeskrankenhausgesetze) lässt sich mit der konkurrierenden Gesetzgebung des Bundes für die vertragsärztliche Bedarfsplanung, von der in §§ 99 - 105 SGB V Gebrauch gemacht hat, somit nicht rechtlich zusammenführen. Die Aufnahme eines Krankenhauses in den Krankenhausplan eines Landes führt zur Zulassung als „Plankrankenhaus“ gem. § 108 Nr. 2 SGB V mit dem Leistungsumfang des § 39 SGB V. Die Zulassung eines Vertragsarztes nach § 95 SGB V führt zur Teilnahmeberechtigung an der vertragsärztlichen Versorgung; die vertragsärztliche Bedarfsplanung begründet Zulassungsbegrenzungen in für Neuzulassungen gesperrten Planungsbereichen, aber deswegen auch die Abhängigkeit der Ermächtigung von Krankenhausärzten/ Krankenhäusern von einer Bedarfsprüfung. Auf verfassungsrechtlicher Grundlage ist die Bedarfsplanung daher sektorenbezogen ausgestaltet (Einschränkung: §§ 115a, 115b, 116b SGB V, DMP).

3.4.4 Daran könnte auch ein gemeinsam auf Landesebene eingerichtetes Koordinierungsgremium nichts ändern. Dessen Entscheidungen könnten nur als Empfehlung in das für den jeweiligen Versorgungsbereich zuständige Gremium eingehen, das sie aber nur insoweit berücksichtigen kann, als es das jeweils geltende Gesetz (SGB V-Landeskrankenhausgesetz) zulässt. Bleibt das SGB V auf die vertragsärztliche Bedarfsplanung begrenzt, ist die Beteiligung von Vertretern der Krankenhausplanung im Landesausschuss bei sektorenübergreifend relevanten Entscheidungen die bessere Alternative.

3.4.5 Je nach Maßgabe einer Gesetzesänderung könnte daher eine gegenüber $\S \S 115 b, 116 b$ SGB V weitergehende Öffnung von Krankenhäusern für die ambulante hoch spezialisierte Versorgung mit entsprechend verschärftem Wettbewerb zur vertragsärztlichen Versorgung erfolgen, diese Öffnung zugunsten eines stärkeren Ausbaus vertragsärztlich strukturierter Schwerpunktpraxen zurückgefahren werden oder der Sicherstellungsauftrag der KÄV auf die hausärztliche und nicht hochspezialisierte fachärztliche Versorgung begrenzt werden. Im geltenden Recht ist eine bundesrechtlich geregelte sektorenübergreifende Bedarfsplanung auf die vertragsärztliche Versorgung begrenzt und muss zu deren Schutz die Einbeziehung von staatlich mitfinanzierten Krankenhäusern grundsätzlich auf einen entsprechend zu begründenden Bedarf abstellen.

3.4.6 Die in den geltenden Bedarfsplanungsrichtlinien verankerte Sonderbedarfszulassung vernachlässigt aber geradezu bewusst das vergleichbare Versor- 
gungsangebot von Krankenhäusern und fördert damit ebenso bewusst die Aufrechterhaltung einer doppelten Facharztschiene in dem besonders kostenintensiven hoch spezialisierten Versorgungsbereich.

\subsection{Regionaler Korrekturfaktor für die Verhältniszahlen}

Die Ausrichtung der vertragsärztlichen Bedarfsplanung an einem durch normativ vorgegebene arztgruppenbezogene Verhältniszahlen definierten allgemeinen bedarfsgerechten Versorgungsgrad kann trotz Unterteilung des Bundesgebietes in neun den Grundsätzen der Raumordnung und Landesplanung entsprechenden Versorgungsebenen bei atypischen Konstellationen zu unsachgemäßen Ergebnissen führen. Deswegen sollte dem Landesausschuss die Zuständigkeit in den Bedarfsplanungsrichtlinien eingeräumt werden, bei derart atypischen Ergebnissen einer Versorgungsanalyse begrenzt auf die regionale Anwendung der Verhältniszahlen auf einen Planungsbereich mit entsprechender Begründung die Verhältniszahl als solche zu modifizieren. Soweit sich aus der Überprüfung des Versorgungsbedarfs für einen Planungsbereich ein grundsätzlicher Mehroder Minderbedarf gegenüber dem allgemeinen bedarfsgerechten Versorgungsgrad ergibt, sollte der Landesausschuss einen Korrekturfaktor beschließen können, der in die örtliche Anwendung der Verhältniszahlen eingeht.

\section{Wahltarife}

Sie stehen neben den Strukturen der vertragsärztlichen Regelversorgung den Krankenkassen als Instrumente des Vertragswettbewerbes, den Versicherten zur Auswahl unter kassenspezifischen Versorgungsangeboten neben der Regelversorgung und den Vertragsärzten als Möglichkeit der Beteiligung an kassenspezifischen Versorgungsangeboten zur Verfügung. Bedarfsplanungsrelevant könnten insbesondere folgende als Wahltarife angebotene Versorgungsformen nach § 53 Abs. 3 SGB V sein

4.1 Integrierte Versorgungsverträge mit Krankenhäusern und Vertragsärzten als Leistungserbringerverbund zu einem sektorenübergreifenden Versorgungsangebot mit der gesetzlich bestehenden Möglichkeit der sektorenübergreifenden Finanzierung durch Bereinigung der Gesamtvergütung und Abzug vom Erlösbudget der Krankenhäuser.
4.2 Hausarzt- und/ oder facharzt-zentrierte Versorgungsangebote mit Vertragsärzten mit intensivierter Koordinierungsverantwortung der in Anspruch genommenen Ärzte auch für Überweisungen, Krankenhauseinweisungen und Verordnungen

4.3 Disease-Managment-Programme (DMP) als ergänzende Versorgungsangebote sowohl zur Regelversorgung als auch zu den aufgezeigten Versorgungsangeboten

Derartige Wahltarife sind kassenspezifische Versorgungsangebote, die keine systematisierte kassenübergreifende und flächendeckende Versorgungsstruktur gewährleistet. Deswegen bleibt eine kassenübergreifende Bedarfsplanung zur Sicherstellung einer flächendeckenden Versorgung notwendig. Insbesondere für regional begrenzte Versorgungsangebote einzelner Krankenkassen gewährleistet die Bedarfsplanung für die Versicherten ein ausreichendes Versorgungsangebot auch außerhalb der gewählten Versorgungsform

\section{Zielsetzung}

Zielsetzung einer Neuordnung der Bedarfsplanung sollte sein:

5.1 stärkere Ausrichtung an den regionalen Strukturen, ohne die zentrale Steuerung durch die bundeseinheitliche Definition des allgemeinen bedarfsgerechten Versorgungsgrades und der Kriterien für regionale Versorgungsanalysen aufzugeben;

5.2 Abbau von Überkapazitäten zugunsten einer gezielten Berücksichtigung eines zusätzlichen Bedarfs an haus- und fachärztlicher Versorgung mit dem Instrument des lokalen Versorgungsbedarfs .

5.3 Abbau von Überkapazitäten in der hochspezialisierten Versorgung durch Einschränkung der doppelten Facharztschiene mit dem veränderten Instrument der Sonderbedarfszulassung

5.4 Bedarfsgerechte Einbindung von Krankenhausangeboten in die vertragsärztliche Bedarfsplanung.

Diese Ziele lassen sich weitgehend mit den bestehenden Instrumenten erreichen, bedürfen jedoch je nach politischer Ausrichtung modifizierter gesetzlicher Vorgaben. 